\title{
Metodología para fomentar el aprendizaje activo de competencias específicas y trasversales a través del soporte de softwares ERPs educativos
}

\author{
Lina Montuori ${ }^{\mathrm{a}}$, Manuel Alcázar-Ortega ${ }^{\mathrm{b}}$, Carlos Vargas-Salgado $^{\mathrm{b}}$ y Paula \\ Bastida-Molina ${ }^{b}$ \\ ${ }^{a}$ Departamento de Termodinámica Aplicada, Universitat Politècnica de València, Camino de Vera, s/n, \\ edificio 5J, 2ª planta.46022 Valencia (España), 1montuori@upvnet.upv.es \\ ${ }^{\mathrm{b}}$ Departamento de Ingeniería Eléctrica, Universitat Politècnica de València, Camino de Vera, s/n, \\ edificio 5E,2 $2^{\mathrm{a}}$ planta. 46022 Valencia (España), malcazar@iiieupv.es, carvarsa@upvnet.upv.es, \\ paubasmo@etsid.upv.es
}

\begin{abstract}
In such a competitive scenario and continuous evolution of university education, it is essential to offer an adequate tool for the development and monitoring of the different competences that students acquire during their training. In this framework, vertical ERP systems, using educational software,, have favored in more than 350 institutions worldwide the creation of a collaborative and learning environment with multiple communication functionalities between professors and students. These management systems allow to build long-term affiliation processes with the possibility of implementing new modules according to the needs. However, although significant advances have been made through its applications, there are still many potentialities that remain unexplored. This article evidences the need to monitor the development of the specific and transversal competences of the students to provide a teaching service that is as close as possible to the particular needs of each student. To achieve this goal, this article proposes a methodology implemented through vertical ERP systems, which favor the dynamic interaction between students and the Academy, as well as the interpretation and understanding of the obtained results.
\end{abstract}

Keywords: Vertical ERP, transversal competences, active learning, diversification and development of talents, methodology, training, evaluation.

\footnotetext{
Resumen

En un escenario competitivo y de evolución continua de la educación universitaria, es fundamental ofrecer una herramienta adecuada para el desarrollo y seguimiento de las diferentes competencias que los alumnos adquieren durante su formación. Los sistemas ERPs verticales, a través del uso de software educativos, han favorecido en más de 350 instituciones a nivel mundial la creación de un entorno de colaboración y aprendizaje con múltiples funcionalidades de comunicación entre profesores y alumnos. Estos
} 
Metodología para fomentar el aprendizaje activo de competencias especificas y trasversales a través del soporte de softwares ERPs educativos

sistemas de gestión permiten construir procesos de afiliación de largo plazo con la posibilidad de implementar nuevos módulos según las necesidades. Sin embargo, aunque se han hecho avances significativos a través de su aplicación, son todavía muchas las potencialidades que permanecen inexploradas. Este articulo evidencia la necesidad de monitorizar el desarrollo de las competencias específicas y trasversales de los alumnos para, de esta manera, proporcionar un servicio de enseñanza que sea lo más cercano posible a las necesidades particulares de cada alumno. Para conseguir este objetivo, este artículo propone una metodología implementada a través de sistemas ERPs verticales, los cuales favorecen la interacción dinámica entre el alumnado y la Academia, así como la interpretación y comprensión de los resultados obtenidos.

Palabras clave: ERP vertical, competencias transversales, aprendizaje activo, diversificación y desarrollo de los talentos, metodología, formación, evaluación.

\section{Introducción}

En el marco actual de avances tecnológicos y transformación digital (Salas Rueda \& Vázquez Estupiñán, 2017), los sistemas ERP (Enterprise Resource Planning - Planificación de Recursos Empresariales) representan una herramienta de información que permite la integración de diferentes operaciones y departamentos de una organización (Andonegi Martínez, et al., 2005). Entre las opciones comerciales disponibles para el sector educativo (Cloud Factory, S.L., 2019), existen varios ejemplos de centros que han adoptado un software ERP vertical para la explotación de distintos módulos de gestión de diferentes áreas del centro (Educación 3.0, 2018). Este tipo de ERP educativo permite personalizar los diferentes módulos y contar con algunos especiales que no poseen otros ERP generales u horizontales (Benvenuto Vera, 2006), (Macías Rodríguez, 2016). De esta manera, los diferentes módulos de estos sistemas de gestión permiten lograr una mayor optimización del trabajo, integrando los diferentes departamentos de la organización universitaria y permitiendo la creación de una base de datos única y común (Huaman Mayta, 2018).

Un sistema ERP diseñado para universidades supone un apoyo esencial en la administración de todas las áreas de gestión, debido a las diferentes funcionalidades de gestión incluidas. Dichas funcionalidades se pueden agrupar en investigación (gestión de propuestas; tablas de gastos e ingresos, proyectos de investigación, etc.), gestión académica (plan de estudios, control de asistencia, notas, etc.) y gestión económica (Cloud Factory, S.L., 2019), (Educación 3.0, 2018).

El presente trabajo se centra en el análisis del módulo de la gestión académica que permita a los profesores y alumnos consultar las calificaciones y gestionar los expedientes. En el marco de este módulo, se propone la implementación de una herramienta de análisis de las calificaciones otorgadas por cada profesor en el apartado de competencias trasversales de cada asignatura. 
Esta herramienta, cuya necesidad ha sido identificada por parte del alumnado durante esta investigación, favorecerá la comunicación entre los alumnos y el profesorado, al tiempo que dará soporte a los estudiantes a la hora de tomar decisiones durante su formación académica. Además, la herramienta proporcionará indicadores de rendimiento universitario, que pueden ayudar a detectar carencias en la formación o prevenir riesgo de abandono.

En el ámbito de la Universidad Politécnica de Valencia, la idoneidad de esta herramienta está justificada por la necesidad de impulsar la integración, en las titulación oficiales, de las competencias trasversales. En particular, esta herramienta puede contribuir a diseñar nuevos procesos de evaluación y acreditación de dichas competencias que sean flexibles e innovadores, dando visibilidad a los resultados adquiridos por los estudiantes tanto para ellos mismos como para la sociedad.

De acuerdo al Plan 2020, el Vicerrectorado de Estudios, Calidad y Acreditación de la UPV está apoyando desde 2012 el proyecto de competencias transversales. Esta iniciativa fue impulsada por el proceso de acreditación de ABET para las titulaciones de Ingeniero Agrónomo, Ingeniero de Caminos, Canales y Puertos, Ingeniero Industrial e Ingeniero de Telecomunicación (Ministerio de Educación, Cultura y Deporte del Gobierno de España, 2017). El resultado de este proceso llevó a la definición trece competencias trasversales, que se enseñan y se trabajan con los alumnos en cualquiera de los títulos oficiales impartidos en la UPV, en cursos de grado y de posgrado (Bonet Espinosa, et al., 2015). Estas competencias representan una innovación significativa, que se espera proporcionen al alumnado una adecuada inserción laboral. Sin embargo, el proceso para su integración sigue en desarrollo, ya que, a diferencia de la competencias específicas, las transversales no aparecen todavía en el título final, por lo que a día de hoy todavía no se ha implementado una metodología apropiada para su control y evaluación (Bonet Espinosa, et al., 2015).

Por último, la herramienta propuesta pretende impulsar, entre otras cosas, el aprendizaje activo y autorregulado por parte de los alumnos. De hecho, según el principio de aprendizaje activo, el estudiante es responsable directo de su aprendizaje y no tiene que limitarse a escuchar el profesor (Oltra Mestre, et al., 2012). Por otro lado, el aprendizaje autorregulado requiere del estudiante que éste perciba sus propias actividades correctamente, evaluando sus resultados y retroalimentando las actividades que sean necesarias (Lamas Rojas, 2008). La metodología propuesta se postula como vía de soporte al desarrollo, control y evaluación de las competencias específicas y transversales, favoreciendo un aprendizaje basado en el establecimiento de metas y en la implicación y motivación constante del alumno.

El artículo se estructura de la siguiente forma: El capítulo 2 presenta los objetivos de la investigación realizada, los cuales se desarrollarán de forma detallada en el capítulo 3, donde se describe la metodología que se ha seguido para el desarrollo de la herramienta propuesta y se definen sus principales funcionalidades. El capítulo 4 muestran los resultados de la encuesta realizada a los estudiantes de grado y un caso práctico de cómo se aplicaría la metodología al Grado en Ingeniería de la Energía impartido en la ETSII de la UPV. Finalmente, las conclusiones del presente trabajo se incluyen en el capítulo 5. 
Metodología para fomentar el aprendizaje activo de competencias especificas y trasversales a través del soporte de softwares ERPs educativos

\section{Objetivos}

Los objetivos en base a los cuales se ha realizado la investigación presentada en este artículo son los siguientes:

- En primer lugar, permitir al profesor realizar un seguimiento particularizado de cada alumno de una manera cómoda, rápida y efectiva, a través del uso de herramientas ERPs.

- Fomentar la interacción entre el profesor y el estudiante, identificando de forma temprana a los alumnos que necesitan fortalecer en mayor medida algunas competencias trasversales específicas.

- Diseñar una metodología que permita el control de variables cualitativas para la gestión de las competencias trasversales, agilizando su interpretación y favoreciendo su desarrollo.

- Ayudar a que el alumno pueda auto-evaluar su proceso de aprendizaje basado en competencias de forma progresiva y continua, al disponer de una herramienta que permita el control de las competencias trasversales

- Finalmente, en base a los objetivos anteriores, sentar las bases para implementar una herramienta que pueda dar soporte al alumno en la elección de asignaturas optativas, así como a elegir sus estudios de postgrado de forma coherente con las competencias alcanzadas, y de acuerdo con sus intereses profesionales.

\section{Desarrollo de la innovación}

\subsection{Contexto académico}

A lo largo de su titulación, los estudiantes de la UPV deben desarrollar una serie de competencias específicas y transversales. Las competencias específicas son las propias del título y están orientadas a la consecución de un perfil específico del egresado (Bonet Espinosa, et al., 2015). Por otro lado, las competencias transversales (denominadas también "key competencies") son competencias transferibles en relación a una variedad de contextos (personales, sociales, académicos y laborales); integradoras, ya que favorecen la formación integral de los estudiantes; interdependientes, ya que trabajando una competencia se desarrollan las dependientes de ella: multifuncionales, debido a su versatilidad como solución a diferentes problemas; $y$ evaluables, ya que se permiten la cuantificación del nivel alcanzado en cada una de ellas por el estudiante.

En el marco del Proyecto de las competencias trasversales mencionado anteriormente, la UPV ha identificado un total trece competencias trasversales, que se resumen en la Tabla 1. Las competencias trasversales son evaluadas a lo largo del curso de estudios en los siguientes momentos:

1. Durante el proceso formativo, a través de asignaturas donde son punto de control

2. Al finalizar los estudios, a través del Trabajo Fin de Grado o Máster.

3. A través de actividades extracurriculares que evidencian el nivel alcanzado en cualquiera de las competencias transversales. 
Tabla 1. Competencias Transversales de la UPV

\begin{tabular}{|c|c|}
\hline Competencia & Descripción \\
\hline 1) Comprensión e integración & $\begin{array}{l}\text { Demostrar la comprensión e integración del } \\
\text { conocimiento tanto de la propia especialización } \\
\text { como en otros contextos más amplios }\end{array}$ \\
\hline 2) Aplicación y pensamiento práctico & $\begin{array}{l}\text { Aplicar los conocimientos teóricos y establecer el } \\
\text { proceso a seguir para alcanzar determinados } \\
\text { objetivos, llevar a cabo experimentos y analizar e } \\
\text { interpretar datos para extraer conclusiones. }\end{array}$ \\
\hline 3) Análisis y resolución de problemas & $\begin{array}{l}\text { Analizar y resolver problemas de forma efectiva, } \\
\text { identificando y definiendo los elementos } \\
\text { significativos que los constituyen. }\end{array}$ \\
\hline 4) Innovación, creatividad y emprendimiento & $\begin{array}{l}\text { Innovar para responder satisfactoriamente y de forma } \\
\text { original a las necesidades y demandas personales, } \\
\text { organizativas y sociales con una actitud emprendedora. }\end{array}$ \\
\hline 5) Diseño y proyecto & $\begin{array}{l}\text { Diseñar, dirigir y evaluar una idea de manera eficaz } \\
\text { hasta concretarla en un proyecto. }\end{array}$ \\
\hline 6) Trabajo en equipo y liderazgo & $\begin{array}{l}\text { Trabajar y liderar equipos de forma efectiva para la } \\
\text { consecución de objetivos comunes, contribuyendo al } \\
\text { desarrollo personal y profesional de los mismos. }\end{array}$ \\
\hline $\begin{array}{l}\text { 7) Responsabilidad ética, medioambiental y } \\
\text { profesional }\end{array}$ & $\begin{array}{l}\text { Actuar con responsabilidad ética, medioambiental y } \\
\text { profesional ante uno mismo y los demás. }\end{array}$ \\
\hline 8) Comunicación efectiva & $\begin{array}{l}\text { Comunicarse de manera efectiva, tanto de forma oral } \\
\text { como escrita, utilizando adecuadamente los recursos } \\
\text { necesarios y adaptándose a las características de la } \\
\text { situación y de la audiencia. }\end{array}$ \\
\hline 9) Pensamiento crítico & $\begin{array}{l}\text { Desarrollar un pensamiento crítico interesándose por } \\
\text { los fundamentos en los que se asientan las ideas, } \\
\text { acciones y juicios, tanto propios como ajenos. }\end{array}$ \\
\hline 10) Conocimiento de problemas contemporáneos & $\begin{array}{l}\text { Identificar e interpretar los problemas } \\
\text { contemporáneos en su campo de especialización, así } \\
\text { como en otros campos del conocimiento. }\end{array}$ \\
\hline 11) Aprendizaje permanente & $\begin{array}{l}\text { Utilizar el aprendizaje de manera estratégica, autónoma } \\
\text { y flexible, a lo largo de toda la vida, en función del } \\
\text { objetivo perseguido. }\end{array}$ \\
\hline 12) Planificación y gestión del tiempo & $\begin{array}{l}\text { Planificar adecuadamente el tiempo disponible y } \\
\text { programar las actividades necesarias para alcanzar } \\
\text { los objetivos, tanto académico-profesionales como } \\
\text { personales. }\end{array}$ \\
\hline 13) Instrumental específica & $\begin{array}{l}\text { Seleccionar y aplicar de forma adecuada las } \\
\text { herramientas, las tecnologías y en general los } \\
\text { instrumentos disponibles para cualquier actuación de } \\
\text { diseño o proyecto relacionados con el ámbito de la } \\
\text { profesión. }\end{array}$ \\
\hline
\end{tabular}

En el marco del presente artículo, la herramienta desarrollada tendrá en cuenta estos tres momentos para la evaluación de las competencias trasversales y fomentará la interacción entre el profesor y alumno a través del uso de una plataforma ERP.

\subsection{Metodología}

La metodología que se ha utilizado para desarrollar la herramienta propuesta para la gestión de competencias transversales se presenta de forma esquemática la Figura 1. En los siguientes apartados de esta sección se explica con mayor detalle cada una de sus fases de desarrollo. 
Metodología para fomentar el aprendizaje activo de competencias especificas y trasversales a través del soporte de softwares ERPs educativos

\subsubsection{Identificación de la necesidad de la herramienta por parte del alumno.}

Para identificar las inquietudes de los alumnos en este aspecto, se ha desarrollado un cuestionario de entrevista personal para preguntar a los estudiantes de forma sistemática sobre sobre la necesidad de una herramienta de soporte en la toma de decisiones y de control del nivel de desarrollo relacionado con las competencias adquiridas a lo largo de sus estudios. Dicho cuestionario, que se incluye en el Anexo I, se ha estructurado en tres apartados:

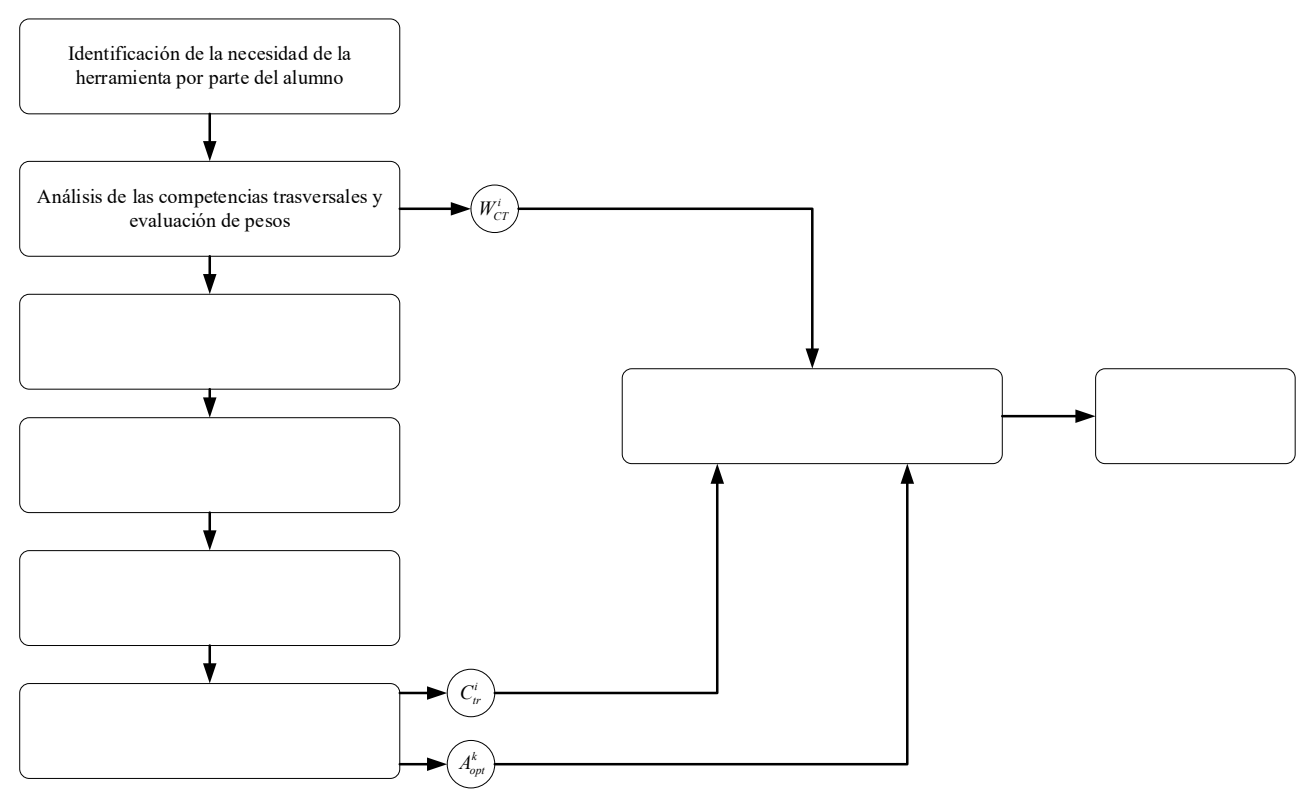

Figura 1. Metodología para el desarrollo de una herramienta ERP para la gestión de competencias

a) Apartado I: Competencias transversales. Se pretende valorar el conocimiento de las competencias transversales por parte del alumnado, su grado de consciencia sobre el estado de desarrollo de este proyecto y la necesidad de una herramienta informática para el soporte en dicho desarrollo.

b) Apartado II: Asignaturas optativas. Se pretende valorar la influencia de las competencias transversales en la elección de las asignaturas optativas por parte del alumno, así como la necesidad de una herramienta informática que le ayude a elegirlas.

c) Apartado III: Postgrado. Se pretende evaluar el grado de incertidumbre de los alumnos hacia su futuro académico y, sobre todo, hacia la elección del curso de postgrado que, en su caso, vayan a seguir. Además, se investiga sobre el grado de necesidad de una herramienta informática para asesorarlos en esta elección.

\subsubsection{Análisis de las competencias trasversales y evaluación de pesos}

El siguiente paso consiste en identificar las competencias transversales que se trabajan en cada una de las asignaturas de la titulación elegida para el curso de grado o de postgrado al 
que se desea aplicar la herramienta. Una vez identificadas, se tendrá que cuantificar el peso de las competencias trabajadas en cada asignatura con respecto al número de créditos de dicha asignatura en la titulación y al número de créditos totales de la titulación. Para ello, se ha definido el índice $W_{C T}^{i}$, que se calcula de la siguiente manera:

$$
W_{C T}^{i}=\frac{\sum_{n=1}^{N} \operatorname{ECTS}_{n}}{\sum_{m=1}^{M} E^{M} T S_{m}} \cdot 100
$$

Donde $W_{C T}^{i}$ es el peso porcentual de la competencia transversal $i$ referido al número total de créditos de la titulacion; $E C T S_{n}$ es el número de créditos de cada una de las $n$ asignaturas en las que se trabaja la competencia transversal $i$; y $E C T S_{m}$ es el número de créditos de cada una de las $m$ asignaturas de la titulación.

\subsubsection{Análisis de las asignaturas optativas}

En esta etapa se identifican las competencias transversales que se trabajan en cada una de las asignaturas optativas de las titulaciones de grado y postgrado consideradas. Asimismo, se analizan las asignaturas previas a cada optativa que el alumno debería haber cursado. El análisis se ha centrado sólo en las asignaturas optativas de tipo técnico, por lo que no se han tenido en cuenta las asignaturas de idiomas o el intercambio académico.

\subsubsection{Definición de los umbrales de desarrollo y acciones correctivas}

De cara a cuantificar numéricamente el nivel de desarrollo de cada competencia, a continuación se ha definido para cada competencia trasversal el rango de variación entre la calificación máxima y mínima alcanzable por el alumno. En la Universidad Politécnica de Valencia, las competencias trasversales son evaluadas a través de rúbricas adoptando una escala cualitativa de 4 valores: A-excelente, B-alcanzada, C-en desarrollo y D-no alcanzada (Bonet Espinosa, et al., 2015). En la Tabla 2 se muestra el valor numérico que se ha asignado a cada uno de estos niveles.

Tabla 2. Escala numérica asignada al nivel de desarrollo de las competencias transversales

\begin{tabular}{lr}
\hline $\begin{array}{l}\text { Nivel de desarrollo } \\
\text { (Escala UPV) }\end{array}$ & $\begin{array}{l}\text { Valor } \\
\text { asignado (GR) }\end{array}$ \\
\hline A-Excelente & 10 \\
B-Adecuado & 8 \\
C-En Desarrollo & 6 \\
D-No alcanzado & 4 \\
\hline
\end{tabular}

En la metodología propuesta, se ha considerado que si el nivel de desarrollo es C o D, se deberán aplicar acciones correctivas para mejorar sólo en aquellas competencias donde se haya obtenido un nivel inferior a B para mejorar el rendimiento de competencias del alumno. Dichas acciones serán propuestas automáticamente por la herramienta, de entre una lista de acciones aplicables en función del tipo de competencia a mejorar. 
Metodología para fomentar el aprendizaje activo de competencias especificas y trasversales a través del soporte de softwares ERPs educativos

\subsubsection{Definición de los tiempos de desarrollo}

Como se indicó en el apartado 3.1, la UPV considera tres momentos en los que las competencias transversales son evaluadas: al final de cada asignatura, al final del TFM o TFG y al final de las actividades extracurriculares. Consecuentemente, se han definido en la metodología los hitos a lo largo del ciclo formativo del alumno en los que las competencias transversales deberían ser evaluadas y cuantificadas:

- Al menos una vez durante los dos primeros cursos del grado, definiéndose un primer "Nivel de Dominio I".

- Una segunda vez en asignaturas punto de control de la franja de tercero y cuarto curso del grado, alcanzando el "Nivel de Dominio II"

- Finalmente, durante la formación del master, llegando hasta un "Nivel de Dominio III".

En la metodología propuesta se ha adoptado un control cuatrimestral durante los dos primeros años del grado, que culmina en alcanzar el Nivel de Domino I. Sucesivamente, a través de otros cuatro controles cuatrimestrales durante el tercer y cuarto año de grado, se llegaría a alcanzar el Nivel de Dominio II, que sería cuando el alumno recibe la primera certificación académica de competencias transversales en el suplemento del título de grado.

Finalmente, los alumnos que continuaran con sus estudios de postgrado, tendrían igualmente un control cuatrimestral del progreso de sus competencias transversales durante uno dos cursos (dependiendo del tipo de máster), pudiendo alcanzar el Nivel de Dominio III, lo que aparecería en el suplemento del título de máster.

La evolución temporal descrita anteriormente se resume en la Figura 2.

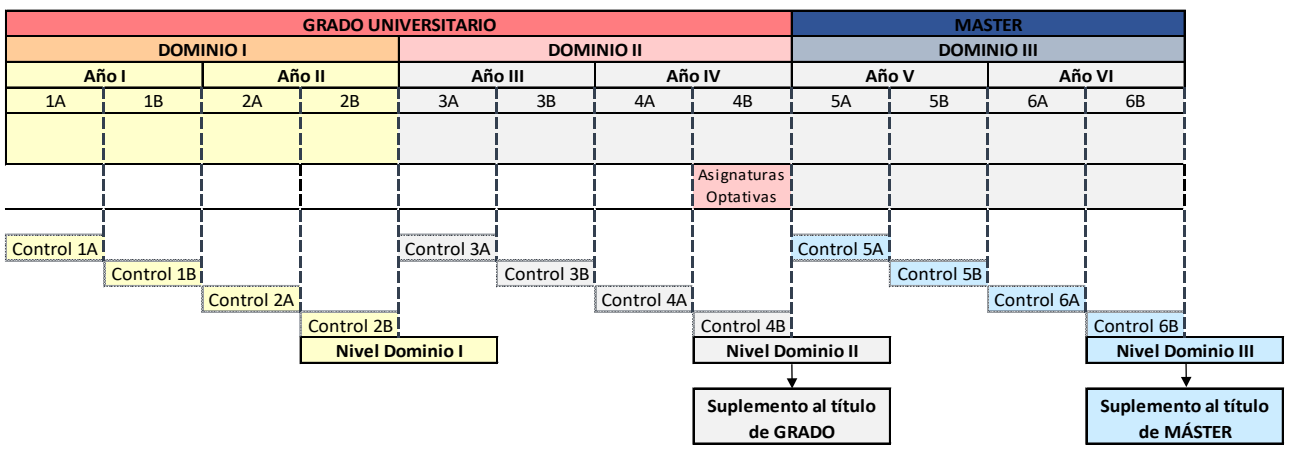

Figura 2. Evolución temporal de la evaluación de competencias transversales en grado y máster

\subsubsection{Definición de los índices de desarrollo}

De forma similar al índice de competencias transversales definido en (1), a continuación se han propuestos tres índices para valorar el nivel de desarrollo alcanzado, el interés técnico y la afinidad del alumno por las asignaturas optativas que éste puede elegir, de cara a sugerirle cuáles podrían ser más adecuadas en su perfil académico. En particular, los índices definidos son los siguientes: 
- Índice de Competencias Transversales $\left(C_{t r}^{i}\right)$. Mediante este índice, el alumno puede obtener una valoración cuantitativa del nivel de desarrollo alcanzado en cada una de las trece competencias transversales. Este índice se define de la siguiente manera:

$$
C_{t r}^{i}=\frac{\sum_{n=1}^{N} \frac{G R_{n}}{10} \cdot \operatorname{ECTS}_{n}}{\sum_{n=1}^{N} E C T S_{n}}
$$

Donde $C_{t r}^{i}$ el índice de la competencia transversal $i ; G R_{n}$ es el grado de desarrollo alcanzado en la asignatura $n$ para la competencia transversal $i$, de acuerdo con la valoración numérica indicada en la Tabla 2; y ECTS $n$ es el número de créditos de cada una de las $n$ asignaturas en las que se trabaja la competencia transversal $i$.

- Índice de Interés Técnico $\left(I^{k}\right.$ tec $)$. Este parámetro mide el interés suscitado en el alumno por cada una de las $k$ asignaturas optativas que puede cursar. Es asignado por el propio alumno mediante la escala Likert, para lo que se propone la gradación incluida en la Tabla 3.

Tabla 3. Escala numérica asignada al Índice de Interés Tecnológico

\begin{tabular}{|l|c|c|c|c|c|}
\hline Escala Likert & $\begin{array}{c}\text { Totalmente } \\
\text { interesado }\end{array}$ & $\begin{array}{c}\text { Muy } \\
\text { interesado }\end{array}$ & $\begin{array}{c}\text { Interés } \\
\text { moderado }\end{array}$ & $\begin{array}{c}\text { Poco } \\
\text { interesado }\end{array}$ & No interesado \\
\hline Escala numérica & 10 & 8 & 6 & 4 & 2 \\
\hline
\end{tabular}

- Índice de Afinidad Tecnológica $\left(A_{\text {opt }}^{k}\right)$. Este parámetro indica la afinidad de los alumnos por las asignaturas optativas que resultarían más idóneas de acuerdo con su perfil, teniendo en cuenta tanto el interés personal que pueda tener por ellas (medido por el índice $I^{k}$ tec) como por el nivel de desarrollo en las competencias transversales relacionadas que el alumno haya alcanzado. Para ello, dicho índice quedaría definido de la siguiente manera:

$$
A_{\text {opt }}^{k}=I_{\text {tec }}^{k} \cdot \frac{\sum_{n=1}^{N}\left(1-\frac{G R_{n}}{10}\right) \cdot \text { ECTS }_{n}}{\sum_{n=1}^{N} E C T S_{n}}
$$

Donde $A_{o p t}^{i}$ es el Índice de Afinidad Tecnológica de la asignatura optativa $k$ a elegir por el estudiante, habiendo sido definidas anteriormente las restantes variables. Como se puede observar, la asignatura optativa $k$ será más idónea para el alumno cuanto mayor sea el valor de este índice.

\subsubsection{Especificación de funcionalidades de la Herramienta de apoyo al alumno}

En base a lo expuesto en los apartados anteriores, en esta sección se definen las principales características que debería tener la herramienta ERP que permitiera, por un lado, informar al alumno sobre el grado de desarrollo de las competencias transversales a lo largo de su carrera; y por otro, aconsejarle en la elección de las asignaturas optativas mediante las que pudiera 
reforzar las competencias con un grado de desarrollo insuficiente y que, a su vez, fueran más acordes con sus intereses académicos. En el caso de la Universidad Politécnica de Valencia, esta herramienta podría estar integrada dentro de la plataforma Poliformat, que ya está vinculada a la base de datos donde se encuentra toda la información relativa a la evaluación de las competencias transversales de los estudiantes y a la que tanto profesores como estudiantes están habituados. La herramienta propuesta debería tener las siguientes funcionalidades:

- Cálculo de los índices definidos en los apartados anteriores.

- Análisis de dichos índices. En particular, la información asociada con cada índice sería la siguiente:

- Dedicación a cada una de las competencias transversales en la titulación. $W_{C T}^{i}$ indicará el peso que en la titulación considerada tiene cada una de las competencias transversales en cuanto al número de ECTS que se dedican para su desarrollo

- Estado actual de desarrollo de cada una de las competencias transversales. $\mathrm{C}_{\mathrm{tr}}^{\mathrm{i}}$ medirá el grado de desarrollo de cada una de las competencias transversales en la formación del alumno, teniendo en cuenta no sólo la calificación alcanzada, sino también los recursos dedicados a cada una a través de los ECTS asignados a cada competencia.

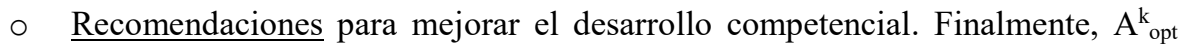
mostrará la idoneidad de las asignaturas optativas que el alumno puede elegir de cara a complementar su formación. Esta información podría mostrarse en forma de ránking, de manera que el alumno pudiera claramente identificar en una jerarquía estandarizada las asignaturas más apropiadas acuerdo con sus intereses.

- Análisis de los resultados de evaluación de las competencias transversales. En caso de que los alumnos no hubieran obtenido una calificación A o B, la herramienta debería intervenir proponiendo algún tipo de acción de mejora correctiva (como la proposición de actividades extracurriculares que los alumnos pudieran realizar de forma complementaria a las asignaturas optativas oficiales)

- Propuesta al alumno sobre las opciones de postgrado. Aunque esta parte no se ha desarrollado completamente en este artículo, la herramienta también deberá proporcionar al alumno una recomendación sobre las titulaciones de postgrado que mejor se adapten a sus intereses, teniendo en cuenta las competencias trabajadas por las asignaturas de cada una de dichas titulaciones, el nivel de desarrollo competencial del alumno y sus intereses particulares, contabilizados a través del índice $\mathrm{I}_{\mathrm{k}}{ }^{\text {tec }}$.

Estas funcionalidades permitirían al alumno monitorizar y cuantificar el nivel de desarrollo de sus competencias transversales a lo largo de todo su proceso formativo y no sólo al final del ciclo, lo que favorecería que el alumno pudiera realizar una auto-evaluación continuada de dicho proceso. Asimismo, estas acciones estarían fomentando un aprendizaje activo por parte del alumno que le permitiría llevar a cabo acciones correctoras para mejorar el nivel de desarrollo de las diferentes competencias transversales trabajadas. 


\section{Resultados}

Este capítulo se divide en dos partes: por un lado, se presentan los resultados obtenidos en la encuesta descrita en el apartado 3.2.1 y cuyo cuestionario se recoge en el Anexo I, donde se analiza el nivel de implantación del proyecto de Competencias Transversales de la UPV y se identifica la necesidad de una herramienta ERP para asesorar a los alumnos a desarrollar adecuadamente dichas competencias. Posteriormente, se presentará un ejemplo donde la metodología descrita en la sección 3.2 se ha aplicado parcialmente a la titulación del Grado de Ingeniería de la Energía que se imparte en la ETSII de la UPV.

\subsection{Resultados de la encuesta a alumnos de grado}

El cuestionario incluido en el Anexo I ha sido utilizado para encuestar a los alumnos de tercer curso de las titulaciones de Grado en Ingenieria Mecánica y Grado en Ingeniería Eléctrica, impartidos por la ETSID de la UPV. La elección de este año de curso se justifica en el hecho de que los alumnos ya han sido evaluados de las competencias trasversales adquiridas a lo largo de tres años, además del hecho de que se encuentran en la tesitura de elegir las asignaturas optativas que complementarán su formación. La encuesta ha sido respondida por una muestra de 92 alumnos de ambos sexos. Las principales conclusiones de la encuesta, agrupadas en los tres apartados mencionados en la sección 3.2.1, son los siguientes:

a) Apartado I: Competencias transversales. Un 65\% de los alumnos encuestados conocen el programa de Competencias Transversales de la UPV y la mayor parte ha sido evaluado en dicho programa. Sin embargo, más de la mitad de los alumnos afirma que no ha sido informado sobre el procedimiento de evaluación ni por el profesor ni por la escuela. Por otro lado, sólo un $51 \%$ de los alumnos encuestados recuerda alguna de las competencias transversales en las que ha sido evaluado, y sólo un $18 \%$ está de acuerdo con la calificación obtenida, mientras que un $76 \%$ ni siquiera recuerda dicha calificación. Con respecto a las competencias transversales en las que los alumnos recuerdan haber sido evaluados se encuentran Pensamiento Crítico (23\%), Trabajo en Equipo (21\%) y Comunicación Efectiva (16\%). El 50\% de los alumnos encuestados opina que el programa de Competencias Transversales le ha ayudado poco o muy poco a desarrollar dichas competencias, mientras que sólo un $13 \%$ opina que ha alcanzado un desarrollo adecuado. En consecuencia, un $90 \%$ de los alumnos encuestados ha indicado que le gustaría disponer de una herramienta de apoyo para el desarrollo de las competencias transversales.

b) Apartado II: Asignaturas optativas. El $84 \%$ de los alumnos encuestados (cursando actualmente $3^{\circ}$ de grado) ya ha elegido las asignaturas optativas que cursará el año próximo. Sin embargo, sólo un $8 \%$ lo ha hecho teniendo en cuenta las competencias que se trabajan en dichas asignaturas de cara a complementar su perfil académico. En este caso, a un $86 \%$ de los encuestados les gustaría ser apoyados por una herramienta en la elección de asignaturas optativas, teniendo en cuenta cómo dichas asignaturas podrían ayudarles a alcanzar un desarrollo adecuado de sus competencias transversales.

c) Apartado III: Postgrado. El $71 \%$ de los alumnos encuestados tiene pensado continuar con estudios de máster cuando terminen el grado que están cursando, aunque sólo un 
$18 \%$ ha elegido ya el máster que realizará. Un $37 \%$ tiene pensado continuar sus estudios de postgrado en la UPV, mientras que un $12 \%$ ya tiene decidido realizarlos en una universidad extranjera. Sin embargo, sólo un $10 \%$ de los encuestados afirma estar satisfecho con la información proporcionada por la Universidad sobre los cursos de postgrados existentes en la UPV. En este sentido, un $98 \%$ de los estudiantes encuestados querría disponer de una herramienta de apoyo que le ayudara a tomar la decisión de qué curso de postgrado encajaría mejor con su formación, competencias adquiridas y expectativas.

\subsection{Caso de aplicación de la metodología al Grado en Ingeniería de la Energía}

La metodología desarrollada ha sido aplicada parcialmente al Grado de Ingeniería de la Energía en la Escuela Técnica Superior de Ingenieros Industriales de la UPV, de acuerdo al plan de estudios 2108-2019. En particular, se ha analizado la dedicación al desarrollo de competencias transversales hasta el primer cuatrimestre del cuarto curso. El Grado en Ingeniería de la Energía se ocupa de la concepción y la gestión de las instalaciones energéticas y sus componentes para garantizar la mejor utilización de los recursos disponibles, aprovechando al máximo las fuentes de energía renovable y minimizando el impacto ambiental. Su oferta anual es de 75 plazas.

Para cuantificar la dedicación al desarrollo de competencias trasversales, de acuerdo con la metodología, se ha determinado qué competencias se trabajan en cada una de las asignaturas del Grado, por cuatrimestre y curso. Una vez identificadas las competencias trasversales, se ha calculado el número de créditos asociado a cada una de ellas durante el período considerado (hasta el $1^{\circ}$ cuatrimestre del cuarto curso inclusive), tal y como se muestra en la Tabla 4. Asimismo, se ha calculado el índice $\mathrm{W}^{\mathrm{i}}$ ст.

Tabla 4. Análisis de competencias transversales del GIE y cálculo del índice $W^{i}{ }_{C T}$

\begin{tabular}{llccc}
\hline Competencia Transversal & $\begin{array}{c}\mathbf{N}^{\mathbf{0}} \\
\text { Asignaturas }\end{array}$ & $\mathbf{E C T S}$ & $\mathbf{W}_{\mathbf{C T}}^{\mathbf{i}}$ \\
\hline 1 & Comprensión e integración & 11 & 64,5 & $30,7 \%$ \\
2 & Aplicación y pensamiento práctico & 13 & 66,0 & $31,4 \%$ \\
3 & Análisis y resolución de problemas & 15 & 78,0 & $37,1 \%$ \\
4 & Innovación, creatividad y emprendimiento & 4 & 19,5 & $9,3 \%$ \\
5 & Diseño y proyecto & 10 & 49,5 & $23,6 \%$ \\
6 & Trabajo en equipo y liderazgo & 7 & 30,0 & $14,3 \%$ \\
7 & Responsabilidad ética, medioambiental y profesional & 5 & 22,5 & $10,7 \%$ \\
8 & Comunicación efectiva & 10 & 49,5 & $23,6 \%$ \\
9 & Pensamiento crítico & 6 & 30,0 & $14,3 \%$ \\
10 & Conocimiento de problemas contemporáneos & 4 & 16,5 & $7,9 \%$ \\
11 & Aprendizaje permanente & 7 & 37,5 & $17,9 \%$ \\
12 & Planificación y gestión del tiempo & 8 & 40,5 & $19,3 \%$ \\
13 & Instrumental específica & 11 & 43,5 & $20,7 \%$ \\
\hline
\end{tabular}


Tal y como muestra la tabla, las competencias trabajadas con mayor dedicación son Análisis y resolución de problemas $\left(\mathrm{W}_{\mathrm{CT}}^{3}=37,1 \%\right)$, Aplicación y pensamiento práctico $\left(\mathrm{W}_{\mathrm{CT}}^{2}=31,4 \%\right)$ y Comprensión e integración $\left(\mathrm{W}^{1} \mathrm{CT}=30,7 \%\right)$. En el lado opuesto, las competencias a las que se dedican menos horas docentes son Conocimiento de problemas contemporáneos $\left(\mathrm{W}^{10} \mathrm{CT}=7,9 \%\right)$, Innovación, creatividad y emprendimiento $\left(\mathrm{W}^{4} \mathrm{CT}=9,3 \%\right)$ y Responsabilidad ética, medioambiental y profesional $\left(\mathrm{W}^{7} \mathrm{CT}^{\mathrm{T}}=10,7 \%\right)$. La Figura 3 muestra las asignaturas optativas que eligen los estudiantes de $4^{\circ}$ curso de GIE en el $2^{\circ}$ semestre junto con las competencias trasversales trabajadas en cada una de ellas. Esta información estaría integrada dentro de la herramienta propuesta y se personalizaría para que el alumno pudiera ver claramente qué asignaturas elegir en función de qué competencias tuviera interés en desarrollar.

\begin{tabular}{|r|l|c|c|c|c|c|c|c|c|c|c|c|c|c|c|}
\hline Código & \multicolumn{1}{|c|}{ Asignatura 4B-OPT } & ECTS & $\mathbf{1}$ & $\mathbf{2}$ & $\mathbf{3}$ & $\mathbf{4}$ & $\mathbf{5}$ & $\mathbf{6}$ & $\mathbf{7}$ & $\mathbf{8}$ & $\mathbf{9}$ & $\mathbf{1 0}$ & $\mathbf{1 1}$ & $\mathbf{1 2}$ & $\mathbf{1 3}$ \\
\hline 12973 & Agua y Energía & 4,5 & & & & & & & & & & & & & \\
\hline 13757 & Applied photochemistry & 4,5 & & & & & & & & & & & & & \\
\hline 12983 & Centrales Nucleares Avanzadas & 4,5 & & & & & & & & & & & & & \\
\hline 12979 & Eficiencia Energética en Edificios & 4,5 & & & & & & & & & & & & & \\
\hline 12971 & Electricidad y sostenibilidad & 4,5 & & & & & & & & & & & & & \\
\hline 12970 & Geotermia & 4,5 & & & & & & & & & & & & & \\
\hline 12969 & Gestión y Planificación Energética & 4,5 & & & & & & & & & & & & & \\
\hline 12974 & $\begin{array}{l}\text { Industrias con alto consumo } \\
\text { energético }\end{array}$ & 4,5 & & & & & & & & & & & & & \\
\hline 12977 & Motores térmicos para automoción & 4,5 & & & & & & & & & & & & & \\
\hline 12982 & Operación de reactores nucleares & 4,5 & & & & & & & & & & & & & \\
\hline 13756 & $\begin{array}{l}\text { Physical concepts in historical and } \\
\text { cultural perspective }\end{array}$ & 4,5 & & & & & & & & & & & & & \\
\hline 12981 & Protección radiológica & 4,5 & & & & & & & & & & & & & \\
\hline 12978 & Química en las Energías renovables & 4,5 & & & & & & & & & & & & & \\
\hline 12980 & Seguridad nuclear & 4,5 & & & & & & & & & & & & & \\
\hline
\end{tabular}

Figura 3. Competencias trasversales trabajadas en las asignaturas optativas de GIE

A partir de este punto, la aplicación de la metodología dependería del perfil particular de cada estudiante. Para aplicar el resto de la metodología, sería necesario calcular los restantes índices aplicados a la calificación particular de los estudiantes, lo cual ha quedado fuera del alcance de esta investigación. No obstante, los autores trabajan en un prototipo de esta herramienta, que será abordado en próximas publicaciones.

\section{Conclusiones}

La investigación que se presenta en este artículo evidencia la necesidad de implementar una herramienta ERP de soporte para ayudar a los alumnos a comprender el estado de desarrollo de sus competencias transversales, así como para ayudarles a, en base a esta información, elegir las asignaturas optativas que mejor complementen su formación. Asimismo, dicha herramienta orientaría a los estudiantes a elegir el curso de postgrado más adecuado para sus intereses académicos y profesionales.

La herramienta propuesta, que en el caso de la UPV podría implementarse como parte de Poliformat, permitiría a los alumnos monitorizar la evolución de sus competencias (no sólo transversales, sino también específicas), detectando en cada momento el estado de desarrollo 
de cada una de ellas, así como aquellas competencias en las que el estudiante debiera mejorar su rendimiento. Además, el alumno podría introducir en la herramienta sus preferencias en cuanto a qué competencias le gustaría desarrollar en particular de cara a complementar su currículo en una dirección determinada. Asimismo, dicha herramienta ayudaría a los profesores a gestionar de una forma más cómoda y sistemática la calificación de los alumnos en base a dichas competencias que, de esta forma, podría incorporarse como un suplemento al título oficial que recibe el alumno al terminar sus estudios de grado o máster.

De cara a sistematizar la monitorización del desarrollo de competencias, este artículo propone una serie de índices de cuantificación, los cuales permiten no sólo determinar si los estudiantes han alcanzado o no ciertas competencias, sino también proponer medidas correctoras de forma automática que les permitan mejorar su desarrollo.

\section{Agradecimientos}

Este trabajo ha sido respaldado en parte por la administración pública de Valencia bajo la beca ACIF/2018/106.

\section{Referencias}

Andonegi Martínez, J. M., Casadesús Fa, M. \& Zamanillo Elguezábal, I., 2005. Evolución histórica de los sistemas ERP: de la gestión de materiales a la empresa digital. Revista de Dirección y Administración de Empresas, Issue 12, pp. 61-72.

Benvenuto Vera, A., 2006. Implementación de sistemas ERP, su impacto en la gestión de la empresa e integración con otras TIC. Capic Review (ISSN 0718-4662), Vol. 4, pp. 33-47.

Bonet Espinosa, P. y otros, 2015. Proyecto Competencias Transversales UPV, Valencia: Universitat Politècnica de València.

Cloud Factory, S.L., 2019. S-ERP Online: Software de gestión para centros educativos, Acceso: 02/03/2019: https://www.conpas.net/sector_educacion.html.

Educación 3.0, 2018. 35 plataformas para la gestión de centros educativos, Acceso: 05/03/2019: https://educaciontrespuntocero.com/novedades2/plataformas-gestionescolar/12663.thml.

Huaman Mayta, D., 2018. Análisis, diseño e implementación del módulo de caja para ERP Educativo de un instituto de idiomas en la ciudad de Huancayo, Huancayo (Perú): Universidad Continental.

Lamas Rojas, H., 2008. Aprendizaje autorregulado, motivación y rendimiento académico. Liberabit. Revista de Psicología (ISSN 1729-4827), Volumen 14, pp. 15-20.

Macías Rodríguez, R. D., 2016. Aplicación ERP orientada a la web para mejorar el control de planificación y gestión educativa de los procesos administrativos circuitales del distrito de educación 23D02 zonal 4 en la ciudad de Santo Domingo, Santo Domingo (Ecuador): Universidad Regional Autónoma de los Andes. 
Ministerio de Educación, Cultura y Deporte del Gobierno de España, 2017. Plan Cultura 2020, Madrid: Secretaría General Técnica, Sub. Gral. de Documentación y Publicaciones.

Oltra Mestre, M. J., García Palao, C., Flor Peris, M. L. \& Boronat Navarro, M., 2012. Aprendizaje activo y desempeño del estudiante: diseño de un curso de dirección de la producción. Working papers on operations management (ISSN 1989-9068), 3(2), pp. 84-102.

Salas Rueda, R. A. \& Vázquez Estupiñán, J. J., 2017. Innovación en el proceso educativo superior a través del servicio en la nube ERPAG. Revista electrónica de calidad en la educación superior (ISSN 1659-4703), 8(2), pp. 62-86.

\section{Anexo I. Encuesta personal}

\begin{tabular}{|c|c|}
\hline Fecha & \\
\hline 1) Indica tu año de curso & Respuesta abierta \\
\hline 2) ¿Estás inscrito en un curso de grado de la UPV? & Respuesta dicótoma \\
\hline 3) ¿Eres un alumno de intercambio? & Respuesta dicotómica \\
\hline
\end{tabular}

\begin{tabular}{|ll|l|}
\hline \multicolumn{2}{|l|}{ Primera Parte: Competencias trasversales } \\
\hline 4$) \quad$ ¿Conoces el programa de competencias transversales de la UPV? & Respuesta dicotómica \\
\hline 5$)$ & $\begin{array}{l}\text { ¿Has sido informado por parte del profesor de la asignatura de las } \\
\text { competencias transversales sobre las que serás evaluado? }\end{array}$ & Respuesta dicotómica \\
\hline 6$)$ & $\begin{array}{l}\text { ¿Has sido informado por parte de la escuela de las competencias } \\
\text { transversales sobre las que serás evaluado? }\end{array}$ & Respuesta dicotómica \\
\hline 7$)$ & $\begin{array}{l}\text { ¿Has sido evaluado este año (primer cuatrimestre) en alguna de las } \\
\text { competencias trasversales? }\end{array}$ & Respuesta dicotómica \\
\hline 8$)$ & $\begin{array}{l}\text { Indicas tres de las competencias trasversales sobre las que ha sido } \\
\text { evaluado este año en el primer cuatrimestre }\end{array}$ & Respuesta abierta \\
\hline 9) & $\begin{array}{l}\text { ¿Estás satisfecho con la valoración recibida en cada una de las } \\
\text { competencias indicadas en la pregunta número 8? }\end{array}$ & Respuesta dicotómica \\
\hline 10) & $\begin{array}{l}\text { Valora cada competencia según su utilidad para tu formación de } \\
\text { cara a tu futuro profesional }\end{array}$ & Escala Likert \\
\hline 11) & Valora cada competencia según tu nivel personal de desarrollo & Escala Likert \\
\hline 12) & $\begin{array}{l}\text { El programa de competencias transversales de la UPV, ¿te ha } \\
\text { ayudado a desarrollar estas competencias? }\end{array}$ & Escala Likert \\
\hline 13) & $\begin{array}{l}\text { ¿Te gustaría disponer de una herramienta en Poliformat que te } \\
\text { ayudara en desarrollar las competencias transversales? }\end{array}$ & Respuesta dicotómica \\
\hline
\end{tabular}

\begin{tabular}{|l|l|}
\hline \multicolumn{2}{|l|}{ Segunda Parte: Asignaturas optativas } \\
\hline 14) ¿Has elegido una asignatura optativa en este curso? & Respuesta dicotómica \\
\hline $\begin{array}{l}\text { 15) } \\
\text { ¿Has tenido en cuenta las competencias trasversales a la hora de } \\
\text { elegir las asignaturas optativas? }\end{array}$ & Respuesta dicotómica \\
\hline $\begin{array}{l}\text { 16) } \\
\text { ¿Te gustaría disponer de una herramienta en Poliformat que te } \\
\text { ayudara en la elección de las asignaturas optativas? }\end{array}$ & Respuesta dicotómica \\
\hline
\end{tabular}

\begin{tabular}{|c|c|}
\hline \multicolumn{2}{|l|}{ Tercera Parte: Postgrado } \\
\hline 17) ¿Has pensado continuar los estudios de máster? & Respuesta dicotómica \\
\hline 18) ¿Vas a continuar tus estudios en la UPV? & Respuesta dicotómica \\
\hline 19) ¿Vas a continuar tus estudios en un centro extranjero? & Respuesta dicotómica \\
\hline $\begin{array}{l}\text { 20) ¿Conoces los diferentes cursos de postgrado que la UPV te } \\
\text { proporciona? }\end{array}$ & Respuesta dicotómica \\
\hline 21) ¿Has elegido en qué curso de postgrado (máster) te inscribirás? & Respuesta dicotómica \\
\hline
\end{tabular}


Metodología para fomentar el aprendizaje activo de competencias especificas y trasversales a través del soporte de softwares ERPs educativos

22) ¿Has sido informado por parte de la escuela de los diferentes cursos de postgrado existentes?

Respuesta dicotómica

23) ¿Está satisfecho con el tipo de asesoramiento proporcionado por la UPV sobre los diferentes cursos de postgrado existentes?

Respuesta dicotómica

24) ¿Te gustaría disponer de una herramienta en Poliformat que te ayudara en la decisión de tu curso de postgrado?

Respuesta dicotómica

(c) Br-NC-ND 2019, Universitat Politècnica de València 